\title{
Ground State of Holographic Mean Field Theory and its Properties in Baryonic Matter
}

Masayasu Harada, Hironori Hoshino*, and Shin Nakamura

Department of Physics, Nagoya University, Nagoya, 464-8602, Japan

E-mail: hhoshino@hken.phys.nagoya-u.ac.jp

We propose a minimal phenomenological extension of the Sakai-Sugimoto model to describe systems of finite baryon density. We introduce two classical Dirac fields with opposite signs of mass terms on the flavor branes so that the theory contains a rotationally invariant state. A preliminary result on the ground state of the model at finite baryon density is also presented.

XV International Conference on Hadron Spectroscopy-Hadron 2013

4-8 November 2013

Nara, Japan

${ }^{*}$ Speaker. 


\section{Introduction}

Quark-hadron physics at finite baryon density is one of the central subjects in modern physics, in connection to studies on neutron stars, quark-gluon plasma and the QCD phase diagram. The AdS/CFT correspondence [1] is a useful method for non-perturbative computations in strongly coupled gauge theories, and the Sakai-Sugimoto model [2] reproduces the properties of low-energy physics of massless large- $N_{c}$ QCD. Recently, there are several proposals to study the finite baryon density system through the holographic QCD models which does not suffer from the sign problem as opposed to the lattice QCD.

However, incorporation of baryons into a holographic model is not straightforward (see, for example, [3). One simple attempt toward a full-fledged model is to describe baryons by a fermionic baryon field on the dual geometry. This idea has been employed in the holographic mean field (HMF) approach in [3] to construct a holographic model of finite baryon density systems.

In the present work, we find that the original model of [3] does not contain rotationally invariant states at finite density. We also point out that the original model is not parity invariant. Then we extend the model in such a way that the model has parity invariance. This can be achieved by introducing two baryon fields into the bulk with opposite signatures of the mass terms. We shall show that the new model contains a rotationally invariant state at finite baryon density. This observation suggests that the idea of parity-doublet model [4] is naturally requested if we follow the holographic mean field approach.

\section{Holographic Mean Field theory and Its Ground State}

In the Holographic Mean Field (HMF) theory proposed by Harada-Nakamura-Takemoto (HNT) in [3], a five dimensional baryonic field is introduced to study the baryonic matters. For demonstrating the idea of HMF approach, HNT used the model given in [5], where the baryon field is introduced into the Sakai-Sugimoto model [2]. The action is given by

$$
\begin{aligned}
S & =S_{\Psi}+S_{A} \\
S_{\Psi} & =\int d x^{4} d w\left[\frac{i}{2} \bar{\Psi} \Gamma^{M} D_{M} \Psi-\frac{i}{2} \overline{\left(D_{M} \Psi\right)} \Gamma^{M} \Psi-m_{5}(w) \bar{\Psi} \Psi\right], \\
S_{A} & =\int d^{4} x d w \mathscr{L}_{A}=-C \int d^{4} x d w U(w)^{1 / 4} \sqrt{\operatorname{det}\left(g_{M N}+\frac{2 \pi \alpha^{\prime}}{N_{C}} F_{M N}\right)},
\end{aligned}
$$

where we have employed a conformally flat metric given by $d s_{5 d}^{2}=H(w)\left(\eta_{\mu \nu} d x^{\mu} d x^{\nu}-d w^{2}\right)$. Here $\eta_{\mu \nu}=\operatorname{diag}(+,-,-,-)$ and $w$ is the radial coordinate on which the boundaries are located at $w= \pm w_{\max }$. The explicit form of $H(w)$ and $U(w)$ are given in [5]. $D_{M}=\partial_{M}-i A_{M}(M=$ $0,1,2,3, w)$ is a covariant derivative, $m_{5}(w)$ denotes the $5 \mathrm{~d}$ mass (see [5] for the explicit form). Here $C=\frac{1}{(2 \pi)^{7}} \frac{4 \pi^{2}}{3} \frac{1}{l_{s}^{1 / 2}}\left(\frac{\lambda}{2 M_{K K}}\right)^{1 / 4} N_{f} N_{C}$, and the $A_{v}(w)$ here is $N_{c}$ times $A_{v}(w)$ in the standard convention. $M_{K K}$ is the energy scale of the theory, $\lambda$ is the ' t Hooft coupling, $N_{f}$ is the number of flavors, and $l_{s}$ is the string length which does not show up in the end of the calculation.

The holographic mean fields are introduced by the following replacement in the above action: $\Psi(x, w) \rightarrow \Psi(w)$ and $A_{M}(x, w) \rightarrow A_{M}(w)$. Equations of motion for the mean fields are

$$
i \Gamma^{w} \partial_{w} \Psi(w)+\Gamma^{M} A_{M}(w) \Psi(w)-m_{5}(w) \Psi(w)=0,
$$




$$
\partial_{w} \frac{\partial \mathscr{L}_{A}}{\partial\left(\partial_{w} A_{N}(w)\right)}-J^{N}(w)=0, \quad J^{N}(w)=\bar{\Psi}(w) \Gamma^{N} \Psi(w) .
$$

It should be noted, although the gauge transformation depending on the $4 \mathrm{~d}$ coordinates has been fixed by the above choice of the mean fields, we still have a residual gauge symmetry in which the gauge transformation depends only on the $w$-coordinate. By using this gauge transformation we take $A_{w}(w)=0$ gauge in the following analysis. Note that equation (2.3) for $N=w$ is $J^{w}(w)=0$. $J^{i}(i=1,2,3)$ may be non-zero in general. We assume that the possible non-zero component is given by $J^{3}$ and we set $J^{1}=J^{2}=0$ without loss of generality. The condition $J^{w}=J^{1}=J^{2}=0$ is realized by taking $\Psi=\left(\Psi_{1}, 0, \Psi_{3}, 0\right)^{T}$ with real $\Psi_{1}$ and $\Psi_{3}$. One can easily confirm that this $\Psi$ with $A_{1}=A_{2}=0$ solves the coupled equations (2.2) and (2.3).

Now the equations of motion (EOM) for $\Psi_{1}$ and $\Psi_{3}$ are

$$
\begin{aligned}
& \left(A_{0}(w)-m_{5}(w)\right) \Psi_{1}(w)+\left(A_{3}(w)+\partial_{w}\right) \Psi_{3}(w)=0, \\
& \left(A_{0}(w)+m_{5}(w)\right) \Psi_{3}(w)+\left(A_{3}(w)-\partial_{w}\right) \Psi_{1}(w)=0 .
\end{aligned}
$$

As we will show in the next section, $A_{\mu}(w)$ transform as $A_{0}(w) \rightarrow A_{0}(-w)$ and $A_{i}(w) \rightarrow-A_{i}(-w)$, under the parity transformation. Using this we decompose the HMF into the parity-even component $A_{\mu}^{(\mathrm{e})}(w)$ and the parity-odd component $A_{\mu}^{(\mathrm{o})}(w)$ as

$$
\begin{array}{llrl}
A_{0}^{(\mathrm{e})}(w) & =\frac{1}{2}\left[A_{0}(w)+A_{0}(-w)\right], & A_{0}^{(\mathrm{o})}(w) & =\frac{1}{2}\left[A_{0}(w)-A_{0}(-w)\right], \\
A_{i}^{(\mathrm{e})}(w) & =\frac{1}{2}\left[A_{i}(w)-A_{i}(-w)\right], & A_{i}^{(\mathrm{o})}(w) & =\frac{1}{2}\left[A_{i}(w)+A_{i}(-w)\right] .
\end{array}
$$

In the present analysis, we assume that $A_{\mu}^{(\text {o })}(w)=0$, i.e., $A_{0}(w)$ is an even function and $A_{i}(w)$ is an odd function of $w$. Then, from (2.4), one can easily see that $\Psi_{1}\left(\Psi_{3}\right)$ is either an even (odd) function of $w$ or an odd (even) function [3].

The physical solution is constrained by the boundary coconditions

$$
\Psi\left( \pm w_{\max }\right)=0, \quad A_{0}\left( \pm w_{\max }\right)=\mu_{0}, \quad A_{3}\left( \pm w_{\max }\right)= \pm \mu_{3},
$$

and by the regularity conditions $\partial_{w} A_{0}(0)=A_{3}(0)=0$. Here $\mu_{0}$ is the baryon chemical potential and $\mu_{3}$ is the source of the axial baryon current. Note that $\Psi\left( \pm w_{\max }\right)=0$ guarantees that the one-point function of the $4 \mathrm{~d}$ fermionic operator associated with $\Psi$ vanishes [6].

We identify the action with the grand potential as discussed in [7, 8, 9: $S=-\int d^{4} x \Omega\left(\mu, \mu_{3}\right)$. Once the solution of EOM is substituted, $S_{\Psi}$ in (2.1) vanishes, while $S_{A}$ is non-zero in which the information of baryons are book-kept. Note that $\Omega$ diverges since the $\mathscr{L}_{A}$ goes infinity at the UV boundaries. Thus we regularize the grand potential $\Omega$ as $\Omega_{r e g}=\Omega-\Omega_{0}$, where $\Omega_{0}$ is the grand potential at zero density limit: $\int d^{4} x \Omega_{0} \equiv-\left.S\right|_{n_{B}=0}$.

In the present analysis we assume that the ground state forms a baryonic matter, which is achieved by taking $\Psi_{1}$ to be an even function with no node. We solve the coupled equations of

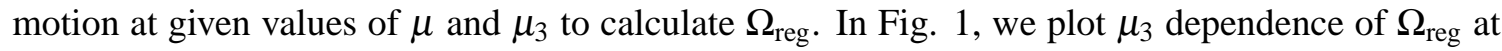
$\mu_{0} / m_{B}=1.01$, where $m_{B}$ is the mass of the ground state baryon. The standard thermodynamics tells that the slope of $\Omega_{\text {reg }}$ gives the expectation value of the axial baryon number current operator conjugate to the source $\mu_{3}$. Figure 11 shows that a non-zero axial current remains even at $\mu_{3}=0$, showing that the rotational invariance is broken. As far as we examined, we could not find any rotationally invariant solution at finite baryon density. 


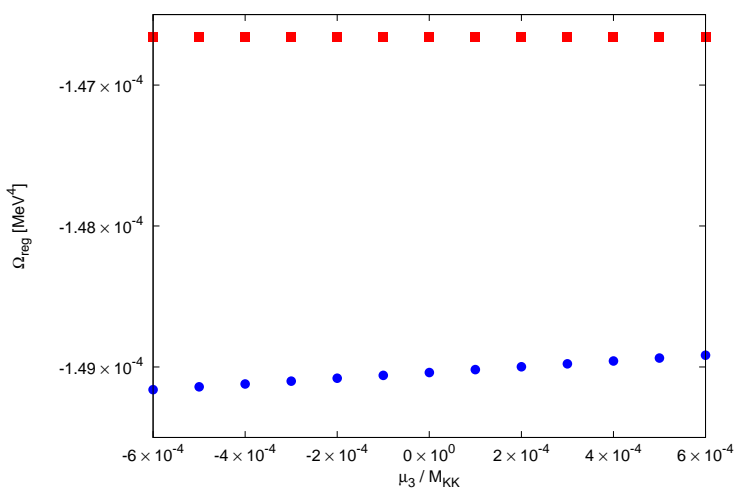

Figure 1: $\Omega_{\text {reg }}$ as a function of $\mu_{3}$ at $\mu / m_{B}=1.01$. The circular plots are shared by both the original model and the extended model whereas the box plots are only for the extended model.

\section{An Extended Model}

Note that the action (2.1) is not constructed in a parity invariant way [10,11]. Let us extend our model to maintain the parity invariance. In order to construct a parity invariant theory, we need to introduce two fermionic fields $\Psi^{\mathrm{I}}$ and $\Psi^{\mathrm{II}}$ with opposite signature of the mass terms [10 11] ${ }^{1}$.

Let us coconsidern action of the baryon sector given by $S_{\Psi}=S_{\Psi}^{\mathrm{I}}+S_{\Psi}^{\mathrm{II}}$, where $S_{\Psi}^{\mathrm{I}}$ is the same as 2.1 with $\Psi$ replaced with $\Psi^{\mathrm{I}}$. The second term is defined as

$$
S_{\Psi}^{\mathrm{II}}=\int d x^{4} d w\left[\frac{i}{2} \overline{\Psi^{\mathrm{II}}} \Gamma^{M} D_{M} \Psi^{\mathrm{II}}-\frac{i}{2} \overline{\left(D_{M} \Psi^{\mathrm{II}}\right)} \Gamma^{M} \Psi^{\mathrm{II}}+m_{5}(w) \overline{\Psi^{\mathrm{II}}} \Psi^{\mathrm{II}}\right] .
$$

Note that $\Psi^{\mathrm{I}}$ and $\Psi^{\mathrm{II}}$ transform as $\Psi^{\mathrm{I}} \rightarrow-\Gamma^{0} \Gamma^{w} \Psi^{\mathrm{II}}$ and $\Psi^{\mathrm{II}} \rightarrow-\Gamma^{0} \Gamma^{w} \Psi^{\mathrm{I}}$ under the parity transformation, and one can confirm that the total action $S_{\Psi}$ is now parity invariant.

In this extended model, the currents $J^{N}(w)$ in (2.3) are replaced with the contributions from $\Psi^{\mathrm{I}}$ and $\Psi^{\mathrm{II}}$ as follows:

$$
J_{N}(w)=J_{N}^{\mathrm{I}}(w)+J_{N}^{\mathrm{II}}(w), \quad J_{N}^{\mathrm{I}, \mathrm{II}}(w)=\overline{\Psi^{\mathrm{I}, \mathrm{II}}}(w) \Gamma^{N} \Psi^{\mathrm{I}, \mathrm{II}}(w) .
$$

We impose the condition $J^{w}=J^{1}=J^{2}=0$ by taking $\Psi^{\mathrm{I}}=\left(\Psi_{1}^{\mathrm{I}}, 0, \Psi_{3}^{\mathrm{I}}, 0\right)^{T}$ and $\Psi^{\mathrm{II}}=\left(\Psi_{1}^{\mathrm{II}}, 0, \Psi_{3}^{\mathrm{II}}, 0\right)^{T}$ with real $\Psi_{1}^{\mathrm{I}, \mathrm{II}}$ and $\Psi_{3}^{\mathrm{I}, \mathrm{II}}$. The equation of motion for $\Psi^{\mathrm{I}}(w)$ is the same as (2.4) whereas the equation of motion for $\Psi^{\mathrm{II}}(w)$ is given by

$$
\begin{aligned}
& \left(A_{0}(w)+m_{5}(w)\right) \Psi_{1}^{\mathrm{II}}(w)+\left(A_{3}(w)+\partial_{w}\right) \Psi_{3}^{\mathrm{II}}(w)=0, \\
& \left(A_{0}(w)-m_{5}(w)\right) \Psi_{3}^{\mathrm{II}}(w)+\left(A_{3}(w)-\partial_{w}\right) \Psi_{1}^{\mathrm{II}}(w)=0 .
\end{aligned}
$$

We should impose the boundary condition $\Psi^{\mathrm{II}}\left( \pm \mathrm{w}_{\max }\right)=0$, on top of the boundary conditions (2.7) and the regularity conditions $\partial_{w} A_{0}(0)=A_{3}(0)=0$ given in the previous section.

The numerical results from the extended model are shown in Fig. 1 The solutions given by the circular plots, which break the rotational invariance at $\mu_{3}=0$, remain solutions in the extended model, whereas new solutions given by boxes appear. We can read the expectation value of the axial current from the slope of the each plots, and we find that the new solutions give vanishing current at $\mu_{3}=0$, restoring the rotational invariance.

\footnotetext{
${ }^{1}$ See also another model [12] based on a bottom-up approach [13].
} 
However the grandpotential of the new solution is greater than the rotationally non-invariant solution. This suggests that the rotational invariance is still broken at zero temperature at small baryon density corresponding to $\mu / m_{B}=1.01$.

\section{Discussions}

We found that the original model proposed by [3] does not have rotationally invariant solution at zero temperature at small baryon density. In the present work, we proposed a parity-invariant extension of the model of [3], and we found that the extended model has a rotationally invariant state. The two baryon fields with opposite parity in the extended model remind us of the parity doublet model [4]. A picture of parity doublet model is naturally requested in the holographic mean field approach.

As far as we have examined within the present work, the rotationally invariant state turned out not to be the ground state, suggesting a spontaneous breaking of the rotational invariance with finite expectation value of the axial current operator. The finite expectation value of axial current operator may be understood as a condensation of axial vector mesons.

However, we postpone a definite conclusion here. The main statement in the present work is to show the presence of a rotationally invariant state in the extended model at finite baryon densities. In order to reach the final conclusion, we think that we should relax the ansatz we have employed, and we need to numerically survey wider range of the possible solutions. These issues remain to be investigated in future work [6].

\section{References}

[1] J. M. Maldacena, Adv. Theor. Math. Phys. 2, 231 (1998); J. M. Maldacena, AIP Conf. Proc. 484, 51 (1999) [Int. J. Theor. Phys. 38, 1113 (1999)], S. S. Gubser, I. R. Klebanov and A. M. Polyakov, Phys. Lett. B 428, 105 (1998), E. Witten, Adv. Theor. Math. Phys. 2, 253 (1998).

[2] T. Sakai and S. Sugimoto, Prog. Theor. Phys. 113, 843 (2005), T. Sakai and S. Sugimoto, Prog. Theor. Phys. 114, 1083 (2005).

[3] M. Harada, S. Nakamura and S. Takemoto, Phys. Rev. D 86, 021901 (2012).

[4] C. E. Detar and T. Kunihiro, Phys. Rev. D 39, 2805 (1989).

[5] D. K. Hong, M. Rho, H. -U. Yee and P. Yi, Phys. Rev. D 76, 061901 (2007). D. K. Hong, M. Rho, H. U. Yee and P. Yi, JHEP 0709, 063 (2007).

[6] M. Harada, H. Hoshino and S. Nakamura, in preparation.

[7] S. Nakamura, Y. Seo, S. -J. Sin and K. P. Yogendran, J. Korean Phys. Soc. 52, 1734 (2008).

[8] K. -Y. Kim, S. -J. Sin and I. Zahed, JHEP 0801, 002 (2008).

[9] S. Kobayashi, D. Mateos, S. Matsuura, R. C. Myers and R. M. Thomson, JHEP 0702, 016 (2007).

[10] K. Shimizu, Prog. Theor. Phys. 74, 610 (1985).

[11] M. Belen Gavela and R. I. Nepomechie, Class. Quant. Grav. 1, L21 (1984).

[12] B. -R. He and M. Harada, Phys. Rev. D 88, 095007 (2013).

[13] D. K. Hong, T. Inami and H. U. Yee, Phys. Lett. B 646, 165 (2007). 